\title{
Optical Coherence Tomography: Clinicopathologic Correlations - The 2016 Gordon K. Klintworth Lecture
}

\author{
Ralph C. Eagle Jr.
}

Department of Pathology, Wills Eye Hospital, Sidney Kimmel Medical College of Thomas Jefferson University, Philadelphia, PA, USA

\section{Keywords \\ Optical coherence tomography · Clinicopathologic correlations · 2016 Gordon K. Klintworth Lecture}

\begin{abstract}
Background/Aims: Spectral domain optical coherence tomography (SD-OCT) has become a mainstay of ophthalmic practice that has revolutionized the assessment and treatment of a variety of ocular disorders. Studies that directly correlate SD-OCT and histopathology are necessary to demonstrate the actual nature of the retinal pathology responsible for the images that resemble histologic sections, but are generated by mathematical algorithms. Methods: Careful correlative light microscopy was performed on a small number of eyes undergoing enucleation for intraocular tumors that had pertinent findings imaged by SD-OCT. Expeditious processing and a fixation technique that prevented retinal detachment minimized tissue distortion and sectioning artifact. Results: The retinal layers disclosed by SD-OCT images do not correspond directly to light microscopy. Photoreceptor ellipsoids can persist in foveal detachments without a distinct ellipsoid band in corresponding SD-OCT images. Lipidized histiocytes can appear as bright hyperreflective spots, and clumps of subretinal macrophages as "shaggy photoreceptors" overlying choroidal tumors. Conclusion:
\end{abstract}

\section{KARGER}

(C) 2017 S. Karger AG, Basel

E-Mail karger@karger.com

www.karger.com/oop
The meaningful interpretation of SD-OCT's striking visual imagery requires a firm foundation in ocular histology and pathology. The accuracy of interpretation can be improved by correlative studies that directly compare OCT and histopathology.

(c) 2017 S. Karger AG, Basel

\section{Introduction}

It is my great honor and privilege to give the International Society of Ophthalmic Pathologists' inaugural Gordon K. Klintworth Lecture at the 2016 World Ophthalmology Congress in Guadalajara, Mexico. I knew Gordon quite well and held him in the highest esteem. He was a scientist and an important basic science researcher as well as an ophthalmic pathologist.

Ophthalmology is a visual pattern recognition specialty. The transparent media of the eye allow retinal pathology to be directly visualized and diagnosed. Imaging has always played a major role in ophthalmic practice, beginning with retinal drawings and paintings and subsequently fundus photography. Our understanding of retinal pathology was further enhanced by the development of intravenous fluorescein angiography, subsequently complemented by indocyanine green angiography. Until recently, in vivo cross- 
sectional imaging of the retina was limited to very-lowresolution images generated by B-scan ultrasonography. Nowadays, optical coherence tomography (OCT) produces high-resolution detailed cross-sectional retinal images that are reminiscent of histologic sections. This relatively new imaging technique has revolutionized the evaluation of retinal disorders and has become the standard of care in the management of age-related maculopathy and diabetic retinopathy in a short period of time. It is also used to assess the optic disc, the nerve fiber layer, and the anterior chamber angle in glaucoma, and is becoming increasingly important in neuro-ophthalmology as well.

OCT uses low-coherence interferometry to produce detailed cross-sectional images of the retina [1]. OCT is a noncontact, noninvasive imaging technique that does not require pupillary dilation or dye injection and hence has no risk of potentially fatal allergic reactions. In addition, the retina is illuminated with near-infrared light, which is invisible to the eye and obviates the need for disturbing bright flashes.

Several varieties of OCT have been used [2]. These include time domain OCT, which was initially employed, and two types of frequency domain OCT. The latter include spatially encoded frequency domain OCT (spectral domain or Fourier domain OCT), which currently is used extensively in many clinics and offices, and swept source OCT (time-encoded frequency domain OCT), which is now commercially available and is being adopted because it has faster scanning and acquisition speeds and a deeper range of imaging.

As noted above, spectral domain OCT (SD-OCT) produces images of the retina that are quite reminiscent of histologic sections. However, the retinal layers disclosed by SD-OCT are generated by mathematical algorithms and do not correspond exactly to the actual retinal layers seen in histologic sections $[3,4]$. These differences are immediately evident when SD-OCT images and histopathology from the same patient are directly correlated (see below). This observation indicates that careful studies that directly correlate histopathology and SD-OCT imaging should be performed, when possible, to ascertain the actual nature of the retinal pathology that appears in SD-OCT images.

\section{A Technique for Processing Eyes to Optimize Correlation}

A few technical challenges must be overcome if SDOCT and histopathology are to be accurately correlated. Artifactitious retinal detachment is a major issue. When unsectioned eyes are fixed routinely by immersion in formaldehyde, artifactitious retinal detachment almost invariably is present in the histologic sections. Optimal correlation between histopathology and SD-OCT requires microscopic sections that preserve anatomic relationships, including attachment of the retina.

A technique designed to prevent retinal detachment was developed in our laboratory. Close and careful communication and cooperation between the pathologist and the ocular oncologist or retinal surgeon who submits the specimen are prerequisites for this technique, which is largely applicable to a small subset of eyes that are enucleated for uveal melanoma. Of course, good-quality SDOCT images of pertinent retinal findings must be performed during the preoperative evaluation of the patient prior to enucleation. Following enucleation, the fresh, unfixed eye is brought expeditiously to the pathology laboratory "while it is still warm." After rapid measurement, the eye is opened coronally through the pars plana or peripheral choroid using one-half of a double-edge razor blade. Macrophotography of pertinent parts of the sectioned globe is performed without immersion in grossing alcohol. Both parts of the eye are then gently immersed in cold $2.5 \%$ glutaraldehyde fixative and refrigerated for $24 \mathrm{~h}$. After fixation, appropriate tissue segments are dissected using fundus photographs and the SD-OCT images as a guide. After macrophotography, the tissue segments are embedded routinely in paraffin and serially sectioned. This fixation technique using glutaraldehyde also would permit electron microscopy. The latter, which would have been advantageous, was not done.

This paper provides illustrative examples of direct clinicopathologic correlation comparing SD-OCT and histopathology from the author's laboratory. It is not meant to be an extensive review of the literature.

\section{Correlation: SD-OCT of Normal Fovea (Fig. 1)}

The eye with a normal fovea shown here had a ciliary body melanoma. The retina in the histologic section (below) remains attached. Comparison of the histopathology (above) and the SD-OCT image (below) graphically illustrate that the layers disclosed by the two techniques do not correlate exactly.

In SD-OCT images, the cellular layers of the retina generally appear as dark bands and the plexiform layers as lighter bands. One of the most striking differences between SD-OCT and histopathology is seen in the outer retinal layers. The correlative histologic sections show 
that the prominent dark band in the outer retina in the SD-OCT image includes more than the outer nuclear layer (ONL). Rather, it includes both the ONL and the outer part of the outer plexiform layer (OPL), which is located external to the middle limiting membrane. That part of the OPL comprises the photoreceptor axons called Henle fibers. (Only the inner part of the OPL located internal to the linear band of synapses called the middle limiting membrane is truly plexiform.) The inner nuclear layer (INL) does appear as a dark band, but the ganglion cell layer, while discernible, is less distinct. The nerve fiber layer of the retina typically appears bright and is more prominent between the fovea and the optic nerve. The latter serves as a tool for orienting OCT images.

\section{The Four Hyperreflective Bands of the Outer Retina} (Fig. 2)

Four hyperreflective bands in the outer retina disclosed by SD-OCT are important landmarks that are used by clinicians to assess the health of the retina and the status of its photoreceptors. A careful and rigorous anatomic study by Spaide and Curcio [3] critically assessed the validity of the anatomic designations commonly used for these bands, and a proposed lexicon of standardized no- menclature was subsequently established by an international consensus panel that included Dr. Spaide [4].

Currently, the four hyperreflective bands are thought to represent (1) the external limiting membrane (XLM) of the retina, (2) the ellipsoid zone of the photoreceptor
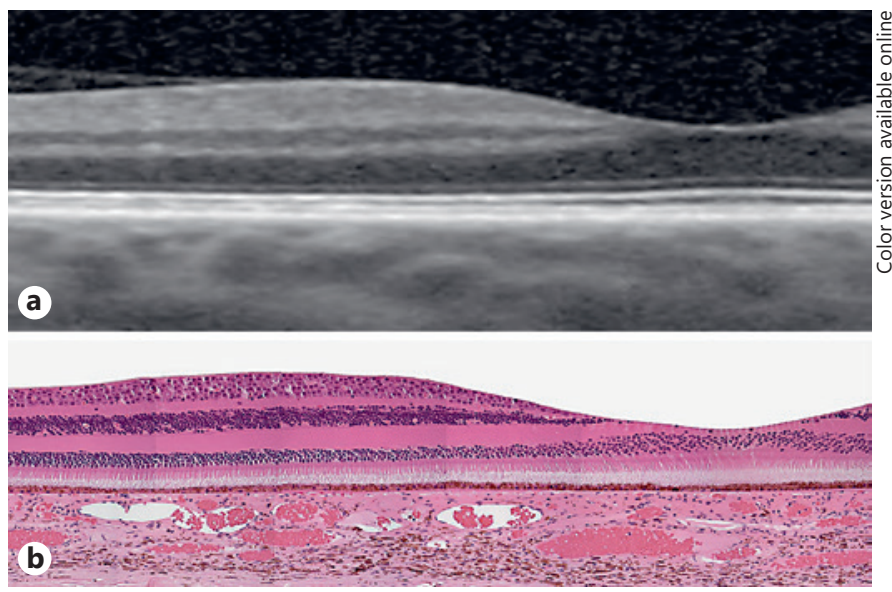

Fig. 1. Normal fovea. a Spectral domain optical coherence tomography (SD-OCT) of normal fovea of an eye enucleated for ciliary body melanoma. b Correlative histopathology of the same eye shows that the retinal layers disclosed by SD-OCT do not correspond exactly with histology. The thick dark outer band in the SDOCT includes the outer nuclear layer and the outer part of the outer plexiform layer. $\mathrm{H} \& \mathrm{E}$ montage, $\times 50$.
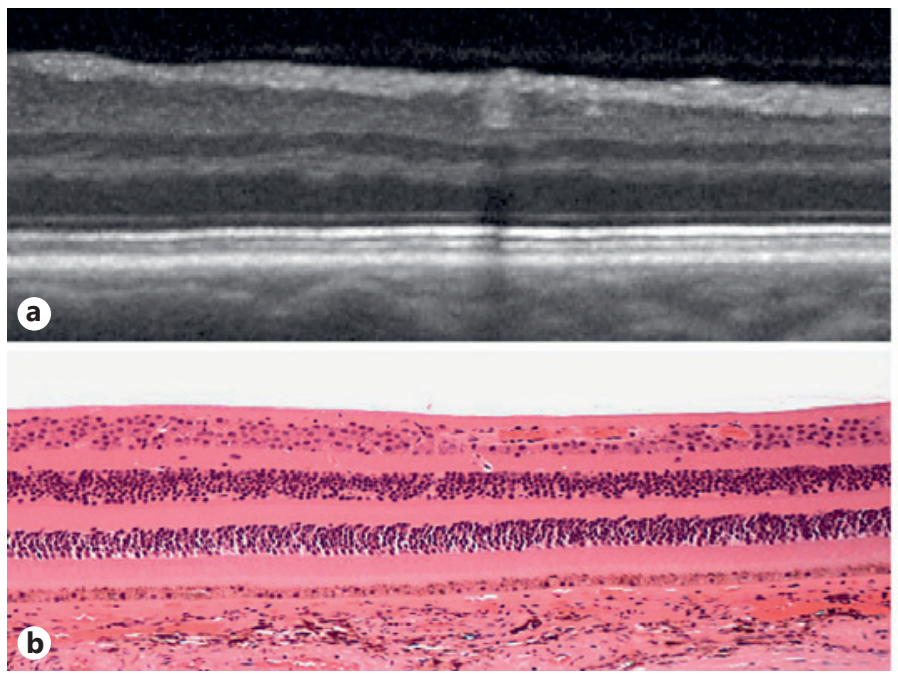

Fig. 2. Spectral domain optical coherence tomography (SD-OCT) of normal retina showing outer hyperreflective bands. a Four hyperreflective bands are seen on the posterior surface of normal retina in SD-OCT. b Histology of the corresponding area in an enucleated eye. $\mathrm{H} \& \mathrm{E}, \times 100$. c Histology of the outer retina. The inner segments of photoreceptors are composed of inner myoid and larger outer ellipsoid zones. H\&E, original magnification
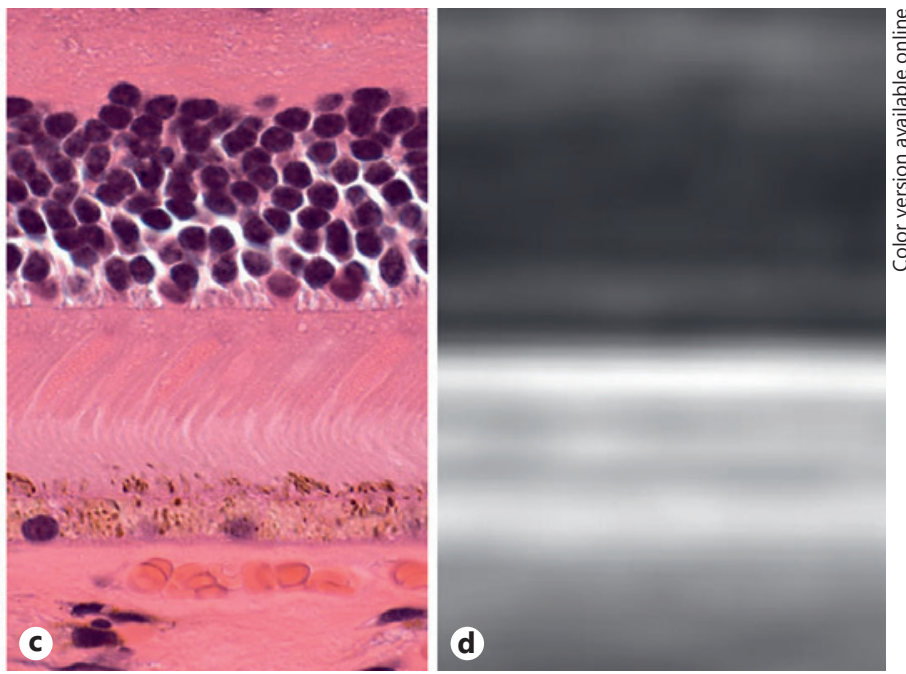

$\times 400$. $\mathbf{d}$ Hyperreflective bands on OCT. The bright inner band on OCT is thought to correspond to a mitochondria-rich ellipsoid layer. The other bands include external limiting membrane, outer segment contact cylinder region, and retinal pigment epithelium choriocapillary complex. A distinct ellipsoid layer serves as a clinical marker for healthy photoreceptors. 
inner segments, (3) the interdigitation zone between the outer segments of the photoreceptors and the apical processes of the retinal pigment epithelium (RPE) that envelope the tips of the outer segments, and (4) the RPE/Bruch membrane complex. In the past, the ellipsoid zone was often termed the photoreceptor inner segment/outer segment junction.

The XLM is a series of intercellular connections that join the apices of the Müller cells and the photoreceptor cells. The inner and outer segments of the photoreceptors project through this belt desmosome into the subretinal space. The inner segments of the photoreceptors have two components: the myoid, which is closest to the XLM, and the ellipsoid. The ellipsoid is packed with mitochondria that provide the energy for the photochemistry of vision. The mitochondria are long and thin and packed in parallel, and their arrangement has been likened to "a bundle of uncooked spaghetti" [3]. The mitochondria may contribute to the bright band on SD-OCT. The interdigitation zone denotes that area where processes on the apical surfaces of the RPE cells envelop the tips of the photoreceptor outer segments. The RPE, the Bruch membrane, and the choriocapillaris probably contribute to the outer band. The histology of the outer retina and the four bands are seen in Figure 2.

Attenuation, discontinuity, or disruption of the XLM, the ellipsoid zone, and the interdigitation zone disclosed by SD-OCT are hallmarks of photoreceptor dysfunction or damage in a variety of retinal diseases. Essentially, the ellipsoid layer is thought to be a good clinical marker for healthy photoreceptors.

\section{The Magnification Factor in SD-OCT Images}

Clinicians who interpret SD-OCT images must recall that the thickness of the retina (vertical axis) is often enhanced or magnified to facilitate interpretation. The magnification factor typically is disclosed by an L-shaped indicator in the margin of the image. In an unmagnified image, the vertical and horizontal lengths of the L-shaped indicator are equivalent. The vertical line is longer in a vertically magnified image, often by a factor of two or three. The effect of image magnification is shown in online supplementary Figure 1S (for all online suppl. material, see www.karger.com/doi/10.1159/000484321). The vertical dimension of the retinal image (above) has been magnified by a factor of three. The unmagnified retinal image is seen below for comparison.

\section{An Eye with Minor Foveal Abnormalities}

SD-OCT and correlative histopathology of an eye with minor foveal abnormalities are seen in online supplementary Figure $2 S$. The patient had a ciliary body melanoma. No clear fundus photographs of the fovea are available, but the quality of the SD-OCT is relatively good. The fovea is attached, but the RPE band in the OCT is focally thickened and there is mild focal diminution of the corresponding ellipsoid layer. The correlative histopathology shows abnormalities in the subfoveal RPE, including cellular budding and thinning of the cells directly beneath the foveola. The other segments of the overlying foveal cones are mildly distorted or indistinct. It should be recalled that foveal cones are somewhat rod-like in their configuration, with thin rod-like inner segments.

\section{A Shallow Perifoveal Detachment in an Eye with a Peripapillary Melanoma}

An eye that was enucleated for a peripapillary melanoma that had a shallow detachment of the fovea is shown in Figure 3. A bright ellipsoid band is seen in the SD-OCT beneath the attached retina at right, but becomes nebulous and indistinct within the area of retinal detachment. The photoreceptors persist in the area of retinal detachment (Fig. 3b), but the ellipsoids of the inner segments appear swollen with a vacuolated appearance. The latter may reflect swelling of the mitochondria. The outer segments also appear swollen and disorderly. Essentially, this correlation shows that a disorderly ellipsoid layer of photoreceptors persists in the area of foveal detachment, but does not produce a bright band in the corresponding OCT.

Online supplementary Figure $3 \mathrm{~S}$ shows at higher magnification how the morphology of the photoreceptors changes within the area of detachment. In the attached part of the retina, the outer segments of the cones are compact and intensely eosinophilic, and only minimal vacuolization is noted in the ellipsoid. As fluid collects in the subretinal space, the ellipsoid layer becomes progressively swollen and vacuolated, and the outer segments become irregular and swollen.

\section{Severe Photoreceptor Loss in an Area of Chronic Retinal Detachment}

It is well recognized that photoreceptors die in a chronically detached retina. Online supplementary Figure $4 \mathrm{~S}$ shows severe photoreceptor atrophy in an area of chron- 
ic retinal detachment. SD-OCT (online suppl. Fig. 4SA) shows thinning of the outer retina with absence of a dark ONL-OPL band and bright outer bands. Correlative histopathology shows total loss of inner and outer segments and severe atrophy of the ONL indicative of photoreceptor cell death. The ONL persists as a disorderly layer of one or two photoreceptor nuclei.

\section{"Shaggy" Photoreceptors and Material in the Subretinal Space Bordering a Choroidal Melanoma}

SD-OCT of shallowly detached perifoveal retina bordering a choroidal melanoma shows a prominent irregular band of material on its posterior surface (online suppl. Figure 5S). The XLM and myoid bands are visible in the SD-OCT, but a distinct ellipsoid layer is not present. The subretinal material contains scattered bright hyperreflective spots. After enucleation, this globe was fixed routinely by immersion in formaldehyde, hence the retina is shallowly detached. Correlative light microscopy shows that the material in the subretinal space is composed of macrophages that contain varying quantities of pigment that includes granules of RPE melanin. Cone nuclei in the ONL are separated from the XLM by a band of cytoplasm, and only the myoid part of the photoreceptor inner segments persists in the subretinal space. The photoreceptors essentially are gone. In this instance, the "shaggy" appearance is caused by a florid collection of macrophages that adhere to the outer retina.

\section{Cystoid Macular Edema in an Eye with a Large Adenocarcinoma of the RPE}

Preoperative OCT of an eye from a young AfricanAmerican man with a large peripheral ciliochoroidal carcinoma of the RPE disclosed cystoid macular edema. SDOCT (online suppl. Fig. 6SA) shows detachment of the fovea by vitreoretinal traction. The posterior hyaloid is detached and a wedge of tissue fills the foveal pit. The eye was enucleated and processed routinely. Fortuitously, routine sections of the artifactitiously detached retina included the fovea (online suppl. Fig. 6SB). Vitreoretinal traction on the epiretinal tissue is no longer evident in the histologic sections. Cystoid spaces corresponding to those seen in the OCT are present in the OPL and the INL. The spaces in the OPL are much larger and contain sparse amounts of granular eosinophilic material.

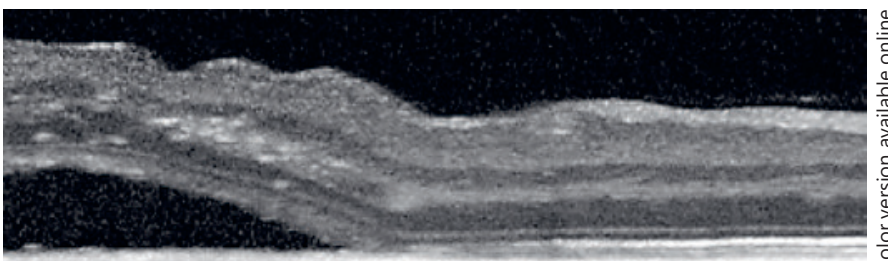

a
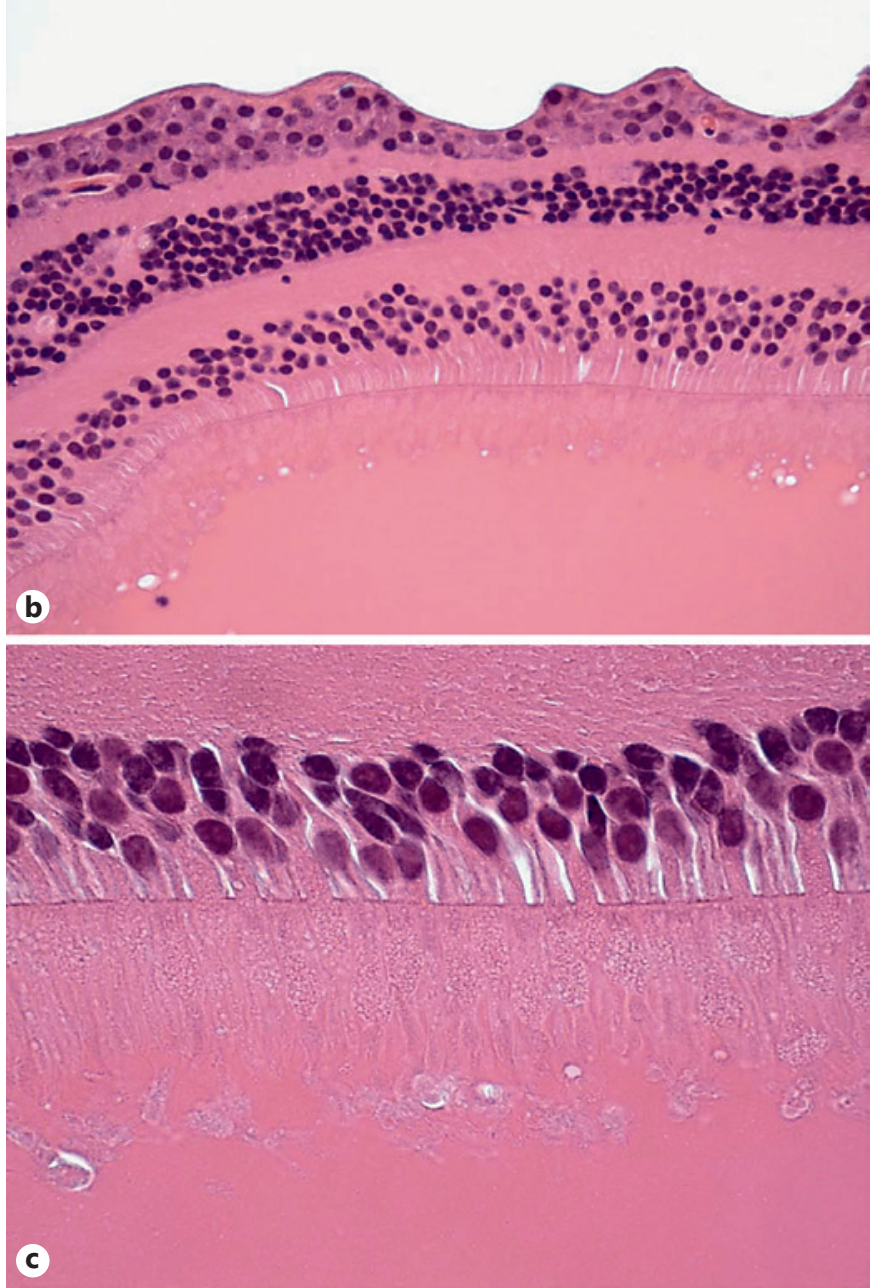

Fig. 3. Foveal detachment: spectral domain optical coherence tomography (SD-OCT) and correlative histopathology. a A bright ellipsoid band area in normal attached retina in SD-OCT at right becomes indistinct in the area of foveal detachment. $\mathbf{b}$ Photoreceptors with ellipsoids persist in the area of foveal detachment. H\&E, $\times 100$. c Photoreceptor ellipsoids in the detached area are swollen, vacuolated, and focally misaligned. H\&E, $\times 400$. 
Cystoid Edema and/or Exudates Overlying Choroidal Melanoma

Online supplementary Figure 7S shows cystoid retinal edema overlying a choroidal melanoma. Cystoid spaces are seen in the OPL of the juxtapapillary retina in the SDOCT image (online suppl. Fig. 7SA). A few smaller spaces are present in the INL. No distinct uniform ellipsoid layer is evident and the RPE appears to be detached. Scattered hyperreflective spots adhere to the outer retina. In the corresponding histopathology, the large cystoid spaces contain pools of proteinaceous fluid instead of the sparse granular fibrinous material typically seen in cystoid macular edema. This densely proteinaceous appearance is more consistent with classic hard exudates. The smaller cysts in the INL appear empty. Despite the absence of a distinct ellipsoid layer, a significant number of photoreceptors persist. There is extensive disruption and detachment of the RPE with segments of denuded Bruch membrane. The planes of sectioning of the two images do not correlate exactly.

\section{Foamy Histiocytes in a Macular Star Figure}

A macular star figure of small yellow hard exudates surrounds the detached fovea in an eye with a peripapillary melanoma (Fig. 4a). SD-OCT discloses an interrupted band of hyperreflective spots in the inner part of the OPL (Fig. 4b). Correlative histopathology (Fig. 4c, d) reveals mononuclear cells with foamy vacuolated cytoplasm consistent with lipidized histiocytes in the OPL and the outer part of the INL. No pools of proteinaceous fluid are evident.

\section{Retinal Exudates at the Margin of a Massively Swollen Optic Disc}

The clinical photograph shows hard exudates in the retina temporal to the massively swollen optic nerve (Fig. 5a). Extraocular extension of a small choroidal melanoma compressed the optic nerve causing severe optic disc edema. SD-OCT (Fig. 5b) shows the massively swollen nerve head. Dark areas resembling cystoid spaces and hyperreflective spots are present in the area of exudation. The hyperreflective spots tend to rim the dark spaces. Correlative histopathology (Fig. 5c) reveals pools of proteinaceous fluid in the OPL and the INL. The spaces are larger in the OPL. Histiocytes with foamy vacuolated cy- toplasm, thought to represent the hyperreflective spots, also are present. A Paton fold is present in the outer retina. The pools of proteinaceous fluid and aggregates of foamy histiocytes are oriented radially by the Henle fibers of the perifoveal retina, forming the macular star (Fig. 5d).

\section{Anterior Segment OCT}

OCT instruments designed specifically to visualize anterior segment structures use a longer wavelength of light that provides better penetration of the sclera [5]. In glaucoma, anterior segment OCT (AS-OCT) is used as an adjunct to gonioscopy to assess anterior chamber angle parameters and narrow angles [6]. In ocular oncology, the technique is used to evaluate tumors and cysts of the iris (online suppl. Fig. 8S), often in conjunction with anterior segment ultrasonography [7]. Corneal specialists use ASOCT to evaluate, diagnose, and manage corneal dystrophies and degenerations $[5,8]$. Online supplementary Figure 9S shows recurrence of granular dystrophy type II (Avellino dystrophy) in the flap after LASIK. Determining the depth of corneal opacities can help to guide ablation in patients undergoing phototherapeutic keratectomy $[5,9]$. In refractive surgery, AS-OCT has been used to detect early signs of forme fruste keratoconus prior to LASIK surgery $[5,10]$. AS-OCT can be used to measure corneal thickness, and AS-OCT measurements of corneal power can increase the accuracy of intraocular lens power calculations in patients who have undergone prior refractive surgery [11]. AS-OCT is also a helpful adjunct in the evaluation and treatment of conjunctival neoplasms including melanocytic lesions and ocular surface squamous neoplasms. AS-OCT provides evidence that melanocytic lesions of the conjunctiva are nevi by detecting the characteristic cysts that are found in two-thirds of cases [12]. Online supplementary Figure 10S shows multiple intralesional cysts disclosed by AS-OCT in an amelanotic compound cystic nevus of the lateral bulbar conjunctiva. Correlative histopathology discloses cystic rests of goblet cell-bearing conjunctival epithelium and an infiltrate of bland amelanotic lymphocytoid nevus cells. High-resolution AS-OCT is used at the Bascom-Palmer eye institute to assess squamous cell lesions of the conjunctiva prior to medical therapy with interferon and other chemotherapeutic agents [13]. Surgical biopsy may still be necessary to exclude invasive squamous cell carcinoma in such cases. Of course, correlative histopathology is more readily available in corneal and conjunctival disease imaged with AS-OCT. 

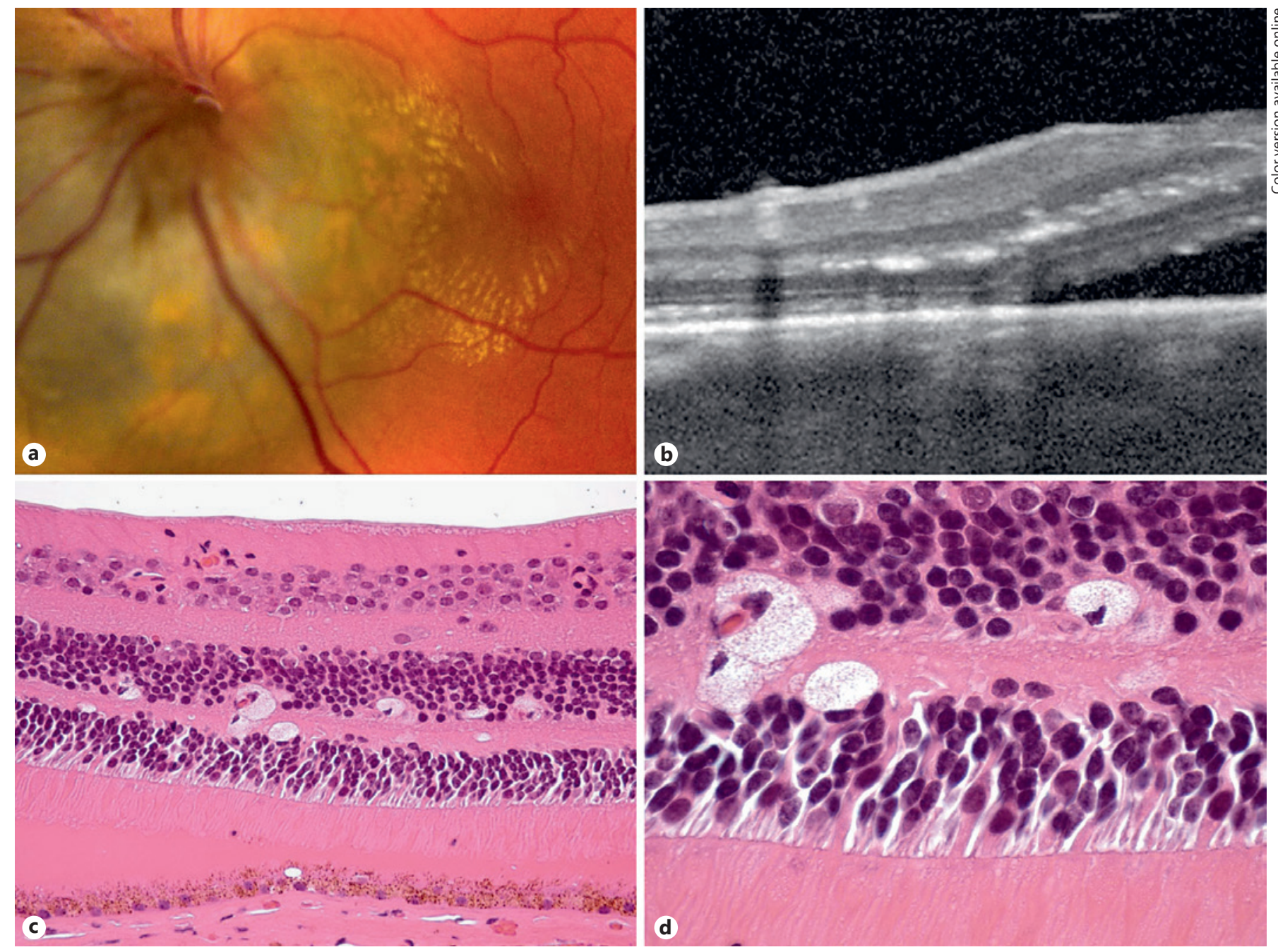

Fig. 4. Lipidized histiocytes comprising hard exudates in macular star figure. a Macular star figure of small yellow hard exudates surrounding the detached fovea in an eye with a peripapillary melanoma. $\mathbf{b}$ An interrupted band of punctate relucences is present in the inner part of the outer plexiform layer in the spectral domain

optical coherence tomography. c, d Correlative histopathology reveals mononuclear cells with foamy vacuolated cytoplasm consistent with lipidized histiocytes in the outer plexiform layer and the outer part of the inner nuclear layer. No pools of proteinaceous fluid are evident. c H\&E, $\times 250$. d H\&E, $\times 400$.

\section{Discussion}

In an astonishingly short period of time, OCT, particularly SD-OCT, has become a mainstay of ophthalmic practice that has revolutionized the evaluation and treatment of a variety of important ocular diseases. Although the in vivo images of ocular anatomy and pathology disclosed by this powerful noninvasive imaging technology are reminiscent of histologic sections, correlative studies demonstrate that the visual data disclosed by these two techniques do not correspond exactly. For example, it is readily evident that the dark band in the outer part of the

retina in SD-OCT images includes more than the ONL of photoreceptor nuclei.

Lacking direct correlation, the interpretation of SDOCT images is based on prior histopathologic studies of retinal pathology. A great deal of speculation and guesswork, which may be inaccurate, is also involved. Studies that directly correlate SD-OCT images and histopathology will increase the accuracy of interpretation by demonstrating the actual nature of the retinal pathology that corresponds to, and is responsible for, the findings in the SD-OCT images. 

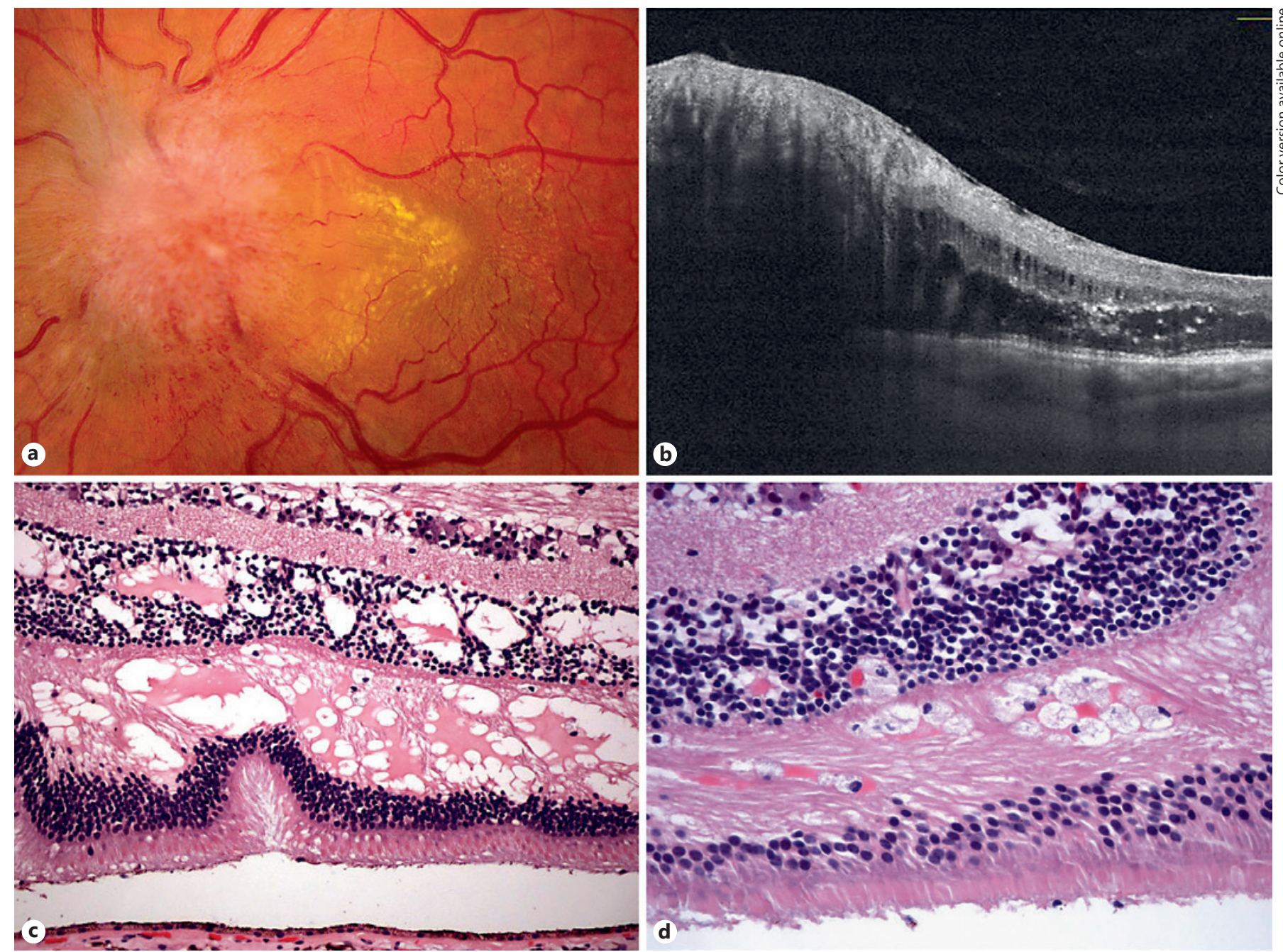

Fig. 5. Retinal exudates adjoining a massively swollen optic disc. a Clinical photograph shows concentric folds and hard exudates in the retinal temporal to massively swollen optic nerve. b Spectral domain optical coherence tomography shows the massively swollen nerve head at left. Dark areas resembling cystoid spaces and punctate relucences are present in the outer retina in the area of exudation. The punctate relucences tend to rim the dark spaces. c Correlative histopathology reveals pools of proteinaceous fluid

In this regard, the findings in several of our correlative studies are somewhat surprising. For example, we have shown that photoreceptors that retain inner segment ellipsoid layers do persist (albeit in a somewhat disorderly state) in areas of foveal detachment that lack a distinct outer retinal ellipsoid band in the corresponding SDOCT. As noted above, a bright outer ellipsoid band generally is thought to be a clinical indicator for healthy photoreceptors. This observation may explain why some pa-

in the outer plexiform and inner nuclear layers. The spaces are larger in the outer plexiform layer. Histiocytes with foamy vacuolated cytoplasm, presumed to correspond to the punctate relucences, are also present. A Paton fold in present in the outer nuclear layer. H\&E, $\times 150$. d The pools of proteinaceous fluid and aggregates of foamy histiocytes are oriented radially by the Henle fibers of the perifoveal retina, forming the macular star. H\&E, $\times 250$.

tients who lack an ellipsoid band on SD-OCT may maintain relatively good visual acuity, and why some are able to reconstitute their ellipsoid band after successful therapy. Our demonstration that lipidized histiocytes correspond to bright hyperreflective spots and hard exudates in some cases is also interesting. Classically, retinal exudates are thought to comprise pools of proteinaceous fluid in the watershed zone of the retina. These new observations suggest that "gitter cells" in isolation can ap- 
pear as hard exudates. Our observations also show that the "shaggy photoreceptors" on the back of the retina overlying choroidal tumors actually may be subretinal macrophages.

It is obvious that additional studies directly correlating SD-OCT and histopathology, preferably employing optimal fixation techniques that maintain retinal attachment and other anatomical relationships, would be highly adventitious. They are necessary to confirm the validity of our preliminary observations. Unfortunately, relatively few eyes are available or suitable for such studies. Enucleated eyes that are candidates for correlative studies must have relatively clear media that permit high-quality OCT. The latter requirement excludes many blind painful eyes that have media opacities. In addition, a significant number of eyes that once were enucleated are now eviscerated. Most of the eyes in this study harbored uveal melanomas. However, only a small proportion of the eyes in that small category actually are available for study because most uveal melanomas currently are treated with eye-sparing plaque brachytherapy. Furthermore, many of the eyes with melanomas that still are enucleated have tumors that are too large to plaque, or contain recurrent tumors that frequently have neovascular glaucoma or vitreous opacities. In both instances, high-quality OCT images suitable for correlative studies usually are not obtainable. Most of the cases in this study contained fairly small peripapillary melanomas that could not be plaqued, or ciliary body tumors that had clear media and attached retinas, an exceedingly small subcategory of cases. The close proximity and cooperation between the Ocular Oncology Service at the Wills Eye Hospital, which treats a high volume of cases, and the ophthalmic pathology laboratory are major factors that led to the accrual of the small number of cases in this study.
The potential for inaccurate and speculative interpretation notwithstanding, OCT is an extremely exciting and powerful imaging modality that has and will continue to shed light on the pathology of retinal disease. OCT undoubtedly will become even more powerful as the technology continues to improve. Many disorders of the retina and choroid have never been examined histopathologically, and most never will. The latter reflects the rarity of many retinal disorders, the paucity of specimens, particularly postmortem eyes, and the dearth of dedicated ophthalmic pathology laboratories that have the appropriate expertise and resources to process and interpret them. This relatively new retinal imaging modality, whose images are so reminiscent of microscopic sections, can provide clues to the basis and pathophysiology of rare disorders of the retina that in all probability will never be examined histopathologically.

It is obvious that the meaningful interpretation of SDOCT's striking visual imagery requires a firm foundation in ocular histology and pathology. Correlative studies that directly compare OCT and histopathology have the potential to increase the accuracy of interpretation.

\section{Statement of Ethics}

This study was approved by the Wills Eye Hospital Institutional Review Board.

\section{Disclosure Statement}

The author has no financial interests or relationships that are relevant to this study.

\section{References}

1 Huang D, Swanson EA, Lin CP, Schuman JS, Stinson WG, Chang W, Hee MR, Flotte T, Gregory K, Puliafito CA, et al: Optical coherence tomography. Science 1991;254:11781181.

2 Podoleanu AG: Optical coherence tomography. J Microsc 2012;247:209-219.

3 Spaide RF, Curcio CA: Anatomical correlates to the bands seen in the outer retina by optical coherence tomography: literature review and model. Retina 2011;31:1609-1619.
4 Staurenghi G, Sadda S, Chakravarthy U, Spaide RF; International Nomenclature for Optical Coherence Tomography (IN.OCT) Panel: Proposed lexicon for anatomic landmarks in normal posterior segment spectraldomain optical coherence tomography: the IN - OCT consensus. Ophthalmology 2014; 121:1572-1578.

5 Ramos JLB, Li Y, Huang D: Clinical and research applications of anterior segment optical coherence tomography - a review. Clin Exp Ophthalmol 2009;37:81-89.
6 Schuman JS: Spectral domain optical coherence tomography for glaucoma (an AOS thesis). Trans Am Ophthalmol Soc 2008; 106 : 426-458.

7 Bianciotto C, Shields CL, Guzman JM, Romanelli-Gobbi M, Mazzuca D Jr, Green WR, Shields JA: Assessment of anterior segment tumors with ultrasound biomicroscopy versus anterior segment optical coherence tomography in 200 cases. Ophthalmology 2011; 118:1297-1302. 
8 Vajzovic LM, Karp CL, Haft P, Shousha MA, Dubovy SR, Hurmeric V, Yoo SH, Wang J: Ultra high-resolution anterior segment optical coherence tomography in the evaluation of anterior corneal dystrophies and degenerations. Ophthalmology 2011;118:1291-1296.

9 Cleary C, Li Y, Tang M, Samy El Gendy NM, Huang D: Predicting transepithelial phototherapeutic keratectomy outcomes using Fourier domain optical coherence tomography. Cornea 2014;33:280-287.
10 Li Y, Meisler DM, Tang M, Lu AT, Thakrar V, Reiser BJ, Huang D: Keratoconus diagnosis with optical coherence tomography pachymetry mapping. Ophthalmology 2008;115: 2159-2166.

11 Huang D, Tang M, Wang L, Zhang X, Armour RL, Gattey DM, Lombardi LH, Koch DD: Optical coherence tomography-based corneal power measurement and intraocular lens power calculation following laser vision correction (an American Ophthalmological Society thesis). Trans Am Ophthalmol Soc 2013;111:34-45.
12 Shields CL, Fasiuddin AF, Mashayekhi A, Shields JA: Conjunctival nevi: clinical features and natural course in 410 consecutive patients. Arch Ophthalmol 2004;122:167-175 erratum in Arch Ophthalmol 2006;124:198.

13 Thomas BJ, Galor A, Nanji AA, El Sayyad F, Wang J, Dubovy SR, Joag MG, Karp CL: Ultra high-resolution anterior segment optical coherence tomography in the diagnosis and management of ocular surface squamous neoplasia. Ocul Surf 2014;12:46-58. 